\title{
Vibration Modelling of Micro Air Vehicle Based on the FW-H Equation
}

\author{
Fu, Li, ${ }^{1}{ }^{*}$, Zhang,Yanwu ${ }^{2, b}$ and Wang,Lingling ${ }^{3, \mathrm{c}}$ \\ ${ }^{1}$ NewBuilding E821, Beihang University, Haidian District, Beijing, China \\ ${ }^{2}$ NewBuilding E822, Beihang University, Haidian District, Beijing, China \\ ${ }^{3}$ NewBuilding E822, Beihang University, Haidian District, Beijing, China \\ achali_fu@yahoo.com, bzywjynxd@sina.com, c147436763@qq.com
}

Keywords: Micro air vehicle; Rotor vibration; FW-H equation; Monopole source.

\begin{abstract}
MAV (Micro air vehicle) has become the most extensive research field in academia and industry because of its strong maneuverability and the capability of VTOL (vertical take-off and landing). Vibration is a key factor affecting the long-term autonomous navigation of MAV, and the dominant source of MAV vibration is the rotor vibration. In order to reduce the impact of rotor vibration on MAV, rotor vibration characteristic of MAV named BHNC-1 developed by our lab is analyzed based on the FW-H (Ffows Williams-Hawkings) equation, and a model of rotor vibration of the BHNC-1 is proposed. The rotor vibration model is validated by comparing the real vibration spectrum and the simulative vibration spectrum. The rotor vibration characteristics of different airfoil are analyzed based on the proposed MAV vibration model, consequently, the optimal installation location of IMU (Inertial Measurement Unit) on the BHNC-1 is seeked out by moving the location of IMU from the middle to the bottom of the BHNC-1.
\end{abstract}

\section{Introduction}

In recent years, as the most active and fastest growing part in the aerospace industry, unmanned aerial vehicle has been playing an increasingly important role in various fields. As an important branch of unmanned aerial vehicle, MAV has a widely application prospect in the field of military and civilian because of its capability of VTOL and the ability to hover and stare ${ }^{[1]}$.

The size of MAV is about $50 \%$ of the small air vehicle, the weight is nearly $20 \%$ of the small air vehicle, and the moment of inertia is $10 \%$ of the small air vehicle. Therefore, the control of MAV is more susceptible to vibration ${ }^{[2,3]}$. Gosiewski ${ }^{[4]}$ has pointed out that the vibration control technology based on the rotor vibration model can effectively reduce the impact of rotor vibration on MAV. Thus, the MAV rotor vibration model for vibration reduction is the basis of flight control of MAV. S. R. Viswamurthy et al. $^{[5]}$ performed the helicopter vibration control using piezoelectric actuated trailing-edge flaps and found the optimal placement of piezoelectric actuated trailing-edge flaps for helicopter vibration control. However there was no analysis and modeling of rotor vibration. In order to research the impact of rotor vibration on the vision system on board, G. Nicolás Marichal Plasencia et al. ${ }^{[6]}$ added the rotor vibration model obtained by simulating the process of unmanned helicopter rotor vibration using modelling software to airframe model. Current research of rotor vibration reduction focuses on the small helicopter rather than MAV. Furthermore, vibration model of helicopter cannot be used on MAV due to the difference of structure. Therefore, it is necessary to find a new method to establish vibration model for MAV. In this paper, a modeling approach to rotor vibration is presented based on the MAV named BHNC-1 developed by our lab. A model of rotor vibration is proposed based on the FW-H equation. Furthermore, the characteristics of rotor vibration of different airfoil are analyzed and the optimal installation location of IMU on the BHNC-1 is seeked out based on the proposed MAV vibration model. Finally, the rotor vibration model is validated.

Rotor vibration model. The BHNC-1 shown in Fig.1 consists of a single propeller, a brushless motor controller and four control vanes attached four fixed stators. The brushless motor rotates constant pitch propeller for mechanical simplicity and changes RPM (Revolutions per Minute) to alter thrust. The fixed stators compensate the yaw moment from reaction torque due to rotor revolution. The 
control vanes work to make moments to control the vehicle attitude in the additional vertical and transitional flight segment. The fixed stators and control vanes are made with carbon-fiber material in order to reduce weight. The benefit of this structure is inherent flight stability with minimum electronics requirement. The control vanes which are immersed in the propeller wash produce control moment under different flight conditions.

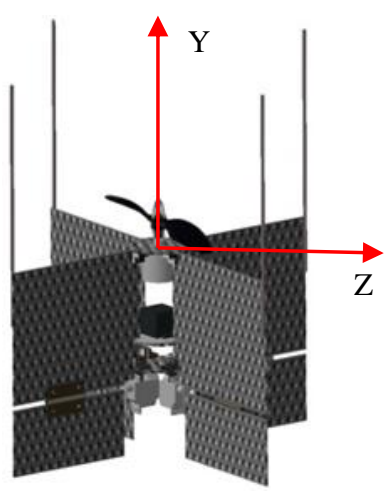

Fig. 1 BHNC-1

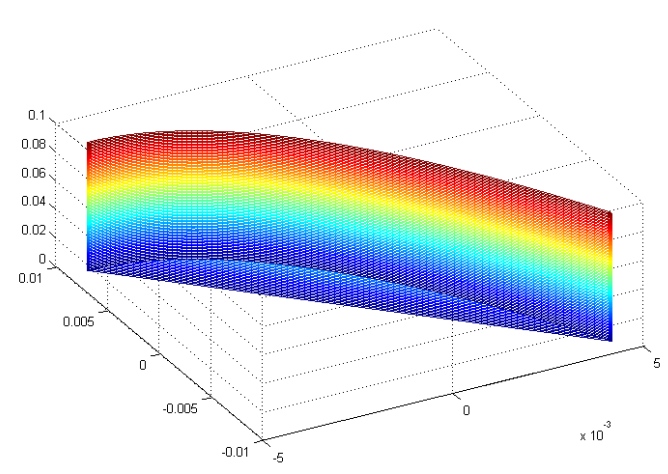

Fig. 2 Mesh on the rotor surface

The dominant source of MAV vibration is the rotor vibration ${ }^{[7]}$. The rotor vibration of MAV is caused by the friction between high speed rotating rotor and air that surrounds it. The periodic expansion-contraction motion of the air induced by rotor vibration can produce monopole ${ }^{[8,9]}$. Hence, rotor vibration model can be obtained by calculating the collection of monopole acoustic pressure.

The origin of the BHNC-1 body coordinate system is placed at the center of the rotor plane, with the positive $\mathrm{Y}$ axis along the longitudinal axis of the body vertically upward, the positive $\mathrm{Z}$ axis perpendicular to the longitudinal axis pointing to the right, as shown in Fig.3. Based on the FW-H equation, monopole acoustic wave equation expressed in the body coordinate system can be written as

$$
\square^{2} p=\frac{\partial}{\partial t}\left[\rho_{0} V_{n}|\nabla \mathrm{f}| \delta(\mathrm{f})\right]
$$

where $\square^{2}$ is D'Alembert wave operator, $p$ is acoustic pressure of rotating rotor, $\rho_{0}$ is air density, $V_{n}$ is normal velocity of rotating rotor, $f$ is equation of rotor surface.

The origin of the BHNC-1 rotor coordinate system is placed at the center of the rotor plane, with the positive $\mathrm{z}$ axis pointing to the right rotor tip, the positive $\mathrm{y}$ axis perpendicular to the rotor plane vertically upward. The initial position of the rotor coordinate system coincide with the body coordinate system.

By transforming $y$ the location of the monopole source in the body coordinate system to $\eta$ the location of the monopole source in the rotor coordinate system and moving the time derivative into the sign of integration, Equation (1) can be expressed as

$$
\begin{aligned}
4 \pi p(\boldsymbol{x}, t) & =\int_{\tilde{\mathrm{f}}=0}\left[\frac{\rho_{0} V_{n}\left(r \dot{M}_{a i} \hat{r}_{i}+c_{0} M_{a r}-c_{0} M_{a}^{2}\right)}{r^{2}\left(1-M_{a r}\right)^{3}}\right]_{r e t} d s \\
& +\int_{\tilde{\mathrm{f}}=0}\left[\frac{\rho_{0} \dot{V}_{n}}{r\left(1-M_{a r}\right)^{2}}\right]_{r e t} d s
\end{aligned}
$$

where $1-M_{a r}$ is Doppler factor, $c_{0}$ is the speed of sound, $\hat{r}_{i}(i=x, y, z)$ is component of $r$ in the rotor coordinate system, $M_{a}$ is Mach number of one of monopole source, $\dot{V}_{n}$ is the normal acceleration of one of monopole source.

The mathematical relationship between the body vibration velocity $u$ caused by rotor vibration and the collection of monopole acoustic pressure $p$ is

$$
u(r, t)=-\frac{1}{\rho_{0}} \int_{-\infty}^{t} \frac{\partial p}{\partial r} \mathrm{~d} \tau
$$

The simultaneous equation of Equation (2) and Equation (3) is the rotor vibration model. 
Model calculation. In order to calculate the acoustic pressure, finite element meshing is performed on rotor surface, each of them can be regard as a monopole source, as shown in Fig.2.

The model calculation steps are

1) Perform finite element meshing, and acquire the data of each mesh;

2) Determine the receiving point, and calculate each monopole source time corresponding to a certain receiving time;

3) Calculate acoustic pressure of each monopole source corresponding to a certain receiving time;

4) Get vibration time-domain waveform and vibration spectrum with the extension of receiving time.

The length of each rotor is $50 \mathrm{~mm}$, and the rotor angular velocity is $510 \mathrm{rad} / \mathrm{s}$. Fig. 3 shows the location of receiving point in the body coordinate system.

The vibration time-domain waveform is obtained as shown in Fig. 4.

Vibration of different airfoil. The characteristics of rotor vibration of different airfoil are analyzed by changing the length of rotor as shown in Fig. 5. Fig. 5 show that the rotor vibration is more intense when the length of the rotor is $100 \mathrm{~mm}$. In the case of adequate lift, the length of the rotor should be shorter.

Vibration of different IMU location. IMU is the core control component of MAV, but it is susceptible to vibration. In order to reduce the influence of rotor vibration on IMU, it is necessary to find a location with minimal vibration. The optimal installation location of IMU on the BHNC-1 is seeked out by moving the location of IMU from $(0,-10 \mathrm{~mm}, 0)$ to $(0,-100 \mathrm{~mm}, 0)$ of the BHNC-1 in the body coordinate system as shown in Fig.6. It is shown that the vibration intensity is minimum at the bottom of the BHNC-1. Thus the optimal location for the IMU should be at the bottom of the BHNC-1.

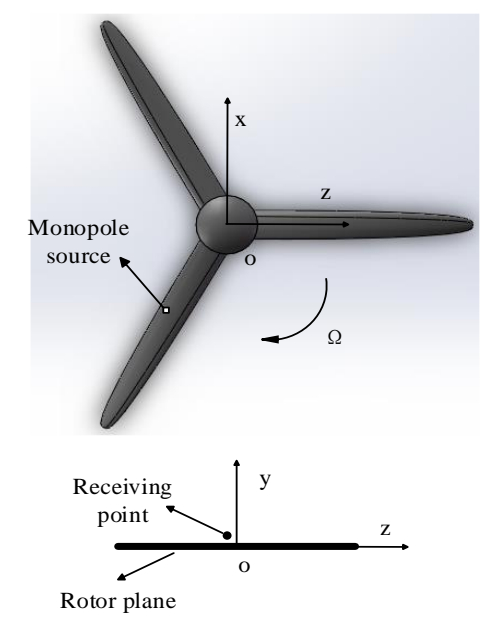

Fig. 3 Monopole source and receiving point

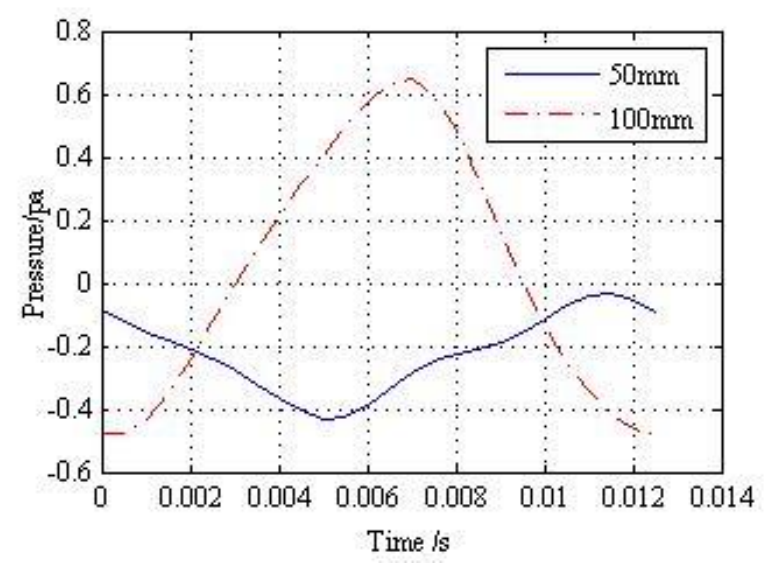

Fig. 5 Vibration of different airfoil

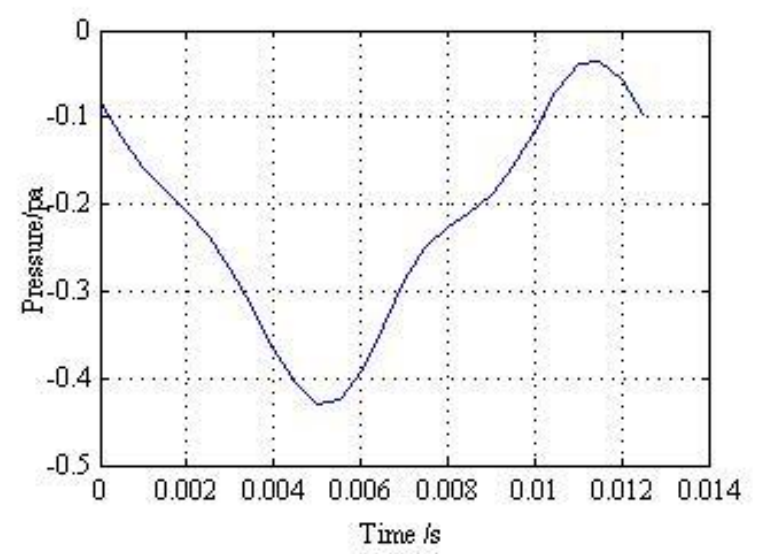

Fig. 4 Calculation result

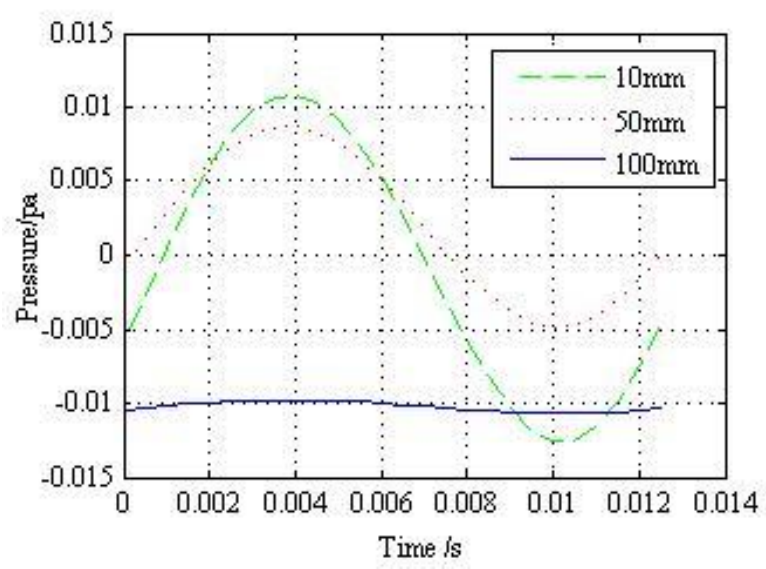

Fig. 6 Vibration of different IMU location 
Model validation. In order to validate the proposed rotor vibration model, the vertical acceleration of IMU on the BHNC-1 in the hover state is collected. The real vibration spectrum of the BHNC-1 is acquired by analyzing measured vertical acceleration data. The simulative vibration spectrum of the BHNC-1 is calculated using the rotor vibration model, as shown in Fig.7.
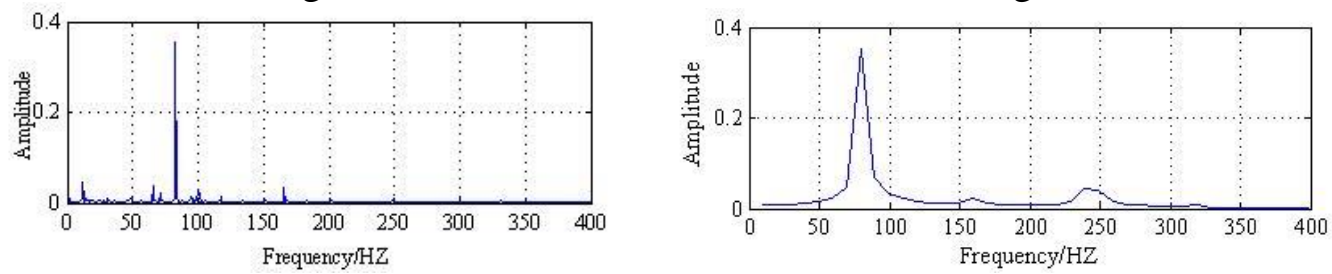

Fig. 7 (left) Real vibration spectrum and, (right) simulative vibration spectrum

Fig.7 shows that both the real vibration dominant frequency and the simulative vibration dominant frequency are approximately $80 \mathrm{~Hz}$. The simulative vibration spectrum is similar to the real vibration spectrum, therefore, the proposed rotor vibration model can be validated.

CONCLUSION. The following conclusions can be obtained

1) The proposed rotor vibration model is validated by comparing the real vibration spectrum and the simulative vibration spectrum;

2) The rotor vibration is more intense with the increase of the rotor length. In the case of adequate lift, the length of the rotor should be shorter;

3) The optimal installation location of IMU on the BHNC-1 is the bottom of the BHNC-1.

\section{References}

[1] Sean George, Paul Samuel. On the design and development of a coaxial nano rotorcraft[C]. AIAA Aerospace Sciences Meeting, 2012, 1:1-24.

[2] Abate G,Stewart K.Babcock J. Autonomous aerodynamic control of Micro Air Vehicle. Air Force Research Laboratory, pp1-72, 2010.

[3] Wang Y. Simultaneous energy harvesting and vibration control via piezoelectric materials. The faculty of the Virginia polytechnic institute and state university, Doctor Thesis, 2012.

[4] Gosiewski, Z. Control-oriented Modeling and control of rotor vibration [J]. Acta Mechanica Automatic, 2008, vol.2, No.2:21-37

[5] Viswamurthy S. R., Ganguli R. Optimal Placement of Piezoelectric Actuated Trailing-Edge Flaps for Helicopter Vibration Control [J]. Journal of Aircraft, 2009, Vol. 46, No. 1:244-253.

[6] G. Nicolás Marichal Plasencia1, María Tomás Rodríguez, Salvador Castillo Rivera2, et al. Modelling and Analysis of Vibrations in a UAV Helicopter with a Vision System [J]. International Journal of Advanced Robotic Systems, 2012, Vol. 9:1-9.

[7] Glaz B, Goel T, Haftka R. Multiple-Surrogate Approach to Helicopter Rotor Blade Vibration Reduction [J].2009, Vol. 47, No. 1:271-282.

[8] Patt D, Li L. Rotorcraft Vibration Reduction and Noise Prediction Using a Unified Aeroelastic Response Simulation [J]. Journal of the American Helicopter Society, 2005, 1:95-106.

[9] Brentner K. Prediction of Helicopter Rotor Discrete Frequency Noise for Three Scale Models [J]. J. AIRCRAFT, 1987, VOL. 25, NO. 5:420-427. 